\title{
Les Mécanismes de protection des droits de l'homme en droit international africain autres que la Cour africaine des droits de l'homme et peuples
}

\author{
Jean-Marc P. MUTONWA KALOMBE
}

\section{INTRODUCTION}

Si le débat autour de l'affirmation de l'existence du droit international africain semble désormais appartenir au passé ${ }^{1}$, c'est autour de sa consolidation qu'entend se concentrer la présente réflexion. Et ce, nonobstant certaines considérations doctrinales fort négativistes qui, comme celle de Blaise TCHIKAYA, « le droit international et donc le droit universel sont insécables et régionalement indivisibles. Au mieux, si un droit international africain codifié devrait exister, il ne le sera qu'au sens de Haya de la Torre $19^{2}{ }_{\gg}$. Or, on le sait, le droit international ne peut être limité au seul aspect conventionnel. Pourtant, renchérit-il, Les particularismes historiques et sociologiques des pays africains deviendraient tout autant des sources d'inspirations normatives en adéquation avec l'ordre universel. La spécificité $\mathrm{du}$ fait international africain s'affirmerait davantage, sans qu'il soit nécessaire de prôner l'existence d'un droit international africain. L'objectif d'universalité invalidant ipso jure une telle existence ${ }^{3}$

En effet, fondé sur ce qu'il est convenu d'appeler le régionalisme africain, le droit international africain répond ainsi d'un système juridique de plus en plus spécifique non seulement s'agissant de ses sources et de ses sujets et/ou acteurs, mais aussi et surtout en ce qui est de ses domaines d'intervention tout comme de ses finalités. De celles-ci, celle touchant à la protection des droits de l'homme semble la plus marquante en ce que ceux-ci font de plus en plus l'objet d'une activité législative au niveau tant continental que régional.

Aussi, certains mécanismes normatifs sont-ils en voie de consolidation dans le cadre du droit de l'Union Africaine alors que des mécanismes institutionnels sont aussi posés dans la visée justement de rendre opérationnelle la protection des droits concernés au travers certaines structures juridictionnelles et /ou quasi juridictionnelles. Tel se dessine le rôle attendu de la Cour Africaine des Droits de l'Homme et des Peuples (CADHP) appelé à se muer, depuis l'avènement du Protocole de Malabo, en la future Cour Africaine de Justice et des Droits de l'Homme (CAJDH).

1 On peut, entre autres auteurs, lire utilement à ce propos les écrits de MAMPUYA, KAZADI MPIANA Joseph, BALINGENE KAHOMBO, NDESHO, BIPOUM.

2 Blaise TCHIKAYA, Les orientations doctrinales de la commission de l'union africaine sur le droit international, in Revue québécoise de droit international, 30.1 (2017), p. 119.

3 Blaise TCHIKAYA, Art. cit., p. 125. 
Par ailleurs et quand bien même telle n'est essentiellement pas leur mission première, les organisations sous-régionales africaines se sont vues, par suite d'une interprétation extensive des actes constitutifs les créant ou de manière explicite, invitées à agir dans le sens de la protection des droits fondamentaux.

Pour ce faire, nous proposons de cerner nos analyses à la fois sur l'indication de ce qu'est, pour l'essentiel, le droit international africain (A), de ce que sont les mécanismes régionaux et sous - régionaux de protection des droits de l'homme en Afrique (B) dont les avancées peuvent être principalement décelées à travers l'action de la Commission Africaine des Droits de l'Homme et des Peuples et mais aussi de celles des structures juridictionnelles régionales et sous-régionales qui, comme dans le cas de la CADHP, appelée à céder l'action à la future $\mathrm{CAJDH}$, mais dont il ne sera pas fait cas dans la présente étude, ont reçu compétence de participer à la concrétisation de ce droit international africain dans sa finalité de garantir la protection des droits de l'homme.

\section{A. A propos du droit international africain}

Lorsqu'au sein de la doctrine l'on analyse la question de l'existence ou non d'un droit international africain, l'on suggère une réflexion autour du fait de savoir si celui-ci, le droit international africain donc, connait une existence autonome à même de conclure à son autonomie juridique. L'idée est celle de savoir si ce droit est constitutif d'un ordre juridique ou d'un système juridique authentiquement autonome de l'ordre juridique international général.

C'est dans cette mesure que Jacques CHEVALLIER ${ }^{4}$ considère que comme ordre, le droit l'est en effet dans les deux sens du terme. Par ordre, selon lui, on peut entendre d'abord l'agencement d'une série d'éléments disparates et hétérogènes en un ensemble cohérent, intelligible : conçu comme synonyme d'ordonnancement, l'ordre désigne alors à la fois le principe logique qui commande les relations entre les divers éléments constitutifs et l'ensemble articulé qu'ils forment. Or, le propre des règles juridiques est précisément qu'elles sont, à l'intérieur d'un même espace social, liées et interdépendantes : " chaque règle de droit est toujours l'élément d'un système, d'un tout, d'un ordre complexe $»^{5}$; elle prend place dans une totalité plus large qui la dépasse et dont elle est tenue de respecter les déterminations et les contraintes ${ }^{6}$.

Mais par ordre, on peut entendre aussi un certain mode d'action et d'emprise sociale : conçu comme synonyme de commandement, l'ordre traduit alors une manifestation d'autorité. Or, la règle de droit s'exprime essentiellement à l'impératif : elle entend obtenir par voie de prescription, d'injonction ou d'interdiction, certains comportements de la part des

4 Jacques CHEVALLIER, L'ordre juridique, sine loci, sine data.

5 G. Gurvitch, L'idée de droit social, Sirey,1932, (rêêd. Scientia 1972), p. 106, cité par Jacques Chevalier, Art. cit., p. 1.

6 Jacques CHEVALIER, Art. cit., p. 1. 
destinataires et la force obligatoire dont elle bénéficie la dote d'une puissance de contrainte irrésistible ${ }^{7}$. Le droit est donc un ordre à la fois en tant qu'il est « systématique » et en tant qu'il est « normatif »; et ces deux aspects, non seulement sont indissolublement liés, mais encore se confortent réciproquement : de même que l'effet normatif du droit est renforcé par la rigueur de sa construction, la dogmatique juridique prend appui sur la force obligatoire de la norme. Ce principe d'ordre, entendu aussi bien sur le plan de l'agencement formel du droit que de son effet social, parait être de l'essence même du phénomène juridique : on le trouve à la base de tous les systèmes juridiques, même s'il a tendance à gagner en rigueur au fur et à mesure de leur développement ${ }^{8}$.

C'est à vrai dire, note l'auteur précité, un élément fondamental du jeu de croyances qui entoure la règle de droit: tandis que son apparence systématique contribue à la parer des attributs de la rationalité, sa puissance normative lui confère le privilège de l'incontestabilité; derrière l'idée d'ordre juridique se profile l'image d'une société organisée, pacifiée et unifiée selon les règles de la raison et en fonction des exigences du bien commun ${ }^{9}$.

Peut-on, tenant compte de ces deux critères, systémique et normatif ${ }^{10}$, caractéristiques d'un ordre juridique, alors soutenir que le droit international africain existe comme constitutif d'un ordre juridique autonome, c'est-à-dire, caractéristique d'un système normatif ayant une identité particulière?

C'est à ce propos que BALINGENE KAHOMBO, dans sa Présentation du Traité de droit international public du professeur Auguste MAMPUYA : Regards croisés sur le régionalisme africain ${ }^{11}$ considère que le régionalisme africain est aussi vieux que le continent africain lui-même. Il suggère que l'on ne doit pas oublier que cette notion, qui n'est pas exclusivement juridique, est avant tout une donnée géographique et historique.

Il réagit ainsi aux écrits du Professeur MAMPUYA KANUNK'a-TSHIABO qui, tout en considérant qu'il existe effectivement un système juridique international africain ou, même,

7 Il faut cependant très vite dissocier la notion de contrainte d'avec celle de sanction s'agissant de l'ordre international ou « des ordres juridiques internationaux ». Lire à ce propos Pierre-Marie DUPUY, P.-M. et KERBRAT, Y., Droit international public, Paris, 13ème éd. Dalloz, 2016.

8 Jacques CHEVALIER note à ce propos que « constatant qu'une intention d'ordre préside à toute œuvre juridique ", S. GOYARD-FABRE y-voit l'expression d'un " besoin d'ordre de la pensée " (p. 273); le droit trouvant son fondement, selon elle, dans " la normativité structurelle de la conscience" (p. 293)», art. cit., p. 2.

9 Idem.

10 Il existe bien d'autres critères dont il faudra tenir compte dans la définition de cette notion d'ordre juridique. Tel pourrait être le cas de la nature de ses sujets, de la spécificité des normes concernées par rapport aux autres normes générales. Mais pour le besoin de la présente, nous entendons nous limiter à ces deux aspects, systémique et normatif, en ce qu'ils nous paraissent suffisant pour répondre à l'interrogation de savoir si le droit international africain est constitutif d'un système juridique particulier ayant secrété ou à même de secrété des normes spécifiquement propres au système juridique concerné.

11 BALINGENE KAHOMBO, Présentation du Traité de droit international public du professeur Auguste Mampuya : Regards croisés sur le régionalisme africain, in Recht in Afrika - Law in Africa - Droit en Afrique, 19 (2016), pp. 209-239. 
un droit international africain, pour reprendre l'expression des plus grands défenseurs de sa spécificité, même si pour lui, soutient pourtant que l'expression est relativiser. Il est toutefois d'avis qu'il y a donc un droit international régional africain, entendu comme un corps des règles établies dans la région pour régir les relations réciproques des membres de la région, ou une « situation » régionale ou, encore, pour reprendre l'expression de l'article 52 de la Charte de l'ONU, une situation qui se prête à une action de caractère régional, africain. Cependant, et pour lui, il n'y a donc rien de surprenant à cela quand on sait que même entre deux Etats seulement un droit international " particulier », local, peut exister dès le moment où il existe entre ces deux Etats un traité ou, ainsi que l'a admis la Cour Internationale de Justice, une coutume locale (Affaire du Droit d'asile) ${ }^{12}$.

C'est que pour lui, le Professeur MAMPUYA donc, l'apparition du système juridique africain et du droit interafricain est un phénomène réel et important de ces cinquante dernières années et par lequel l'Afrique participe à l'élaboration du droit international, décolonisant du même coup le processus de formation des normes internationales en Afrique. Pourtant, ce qui est d'évidence une contradiction, ce droit interafricain, selon lui, tout particulier qu'il soit, est loin d'être un droit spécifique ${ }^{13}$.

Et pourtant, il faut l'admettre avec BALINGENE KAHOMBO, le régionalisme africain, est aussi politique, économique, idéologique, social et culturel.

Sur le plan juridique, il comporte, comme tout régionalisme de ce type, trois acceptions distinctes, à savoir un ensemble d'approches ou de méthodes spécifiques d'appréhender ou d'interpréter le droit international ${ }^{14}$, une approche régionale d'élaboration des normes de droit international, lesquelles peuvent s'étendre progressivement au niveau global, et une exception géographique aux règles universelles de droit international, l'application des normes adoptées restant limitée aux Etats concernés de la région ${ }^{15}$.

Son développement contemporain est lié à l'histoire de la construction de l'unité africaine, de l'émancipation du continent africain, et adossé à l'idéologie panafricaine, dont les origines remontent au 19ème siècle au sein de la diaspora noire aux Antilles et en Amérique du Nord.

Néanmoins, le régionalisme juridique africain ne s'est véritablement cristallisé, de l'avis de cet auteur, qu'avec l'avènement des Etats indépendants en Afrique, l'organisation des conférences panafricaines à Accra (Ghana) en 1958 et la création de premières organi-

12 MAMPUYA KANUNK'a-TSHIABO, Traité de droit international public, MEDIASPAUL, KINSHASA, 2016, pp. 151-152.

13 Idem, p. 153.

14 Voir Jon Woronoff, Organizing African Unity, Metuchen 1970; Van Chien Nguyen, Les politiques d'unité africaine, thèse de 3e cycle, Université de Paris V 1973; Oswald Ndeshyo, L'héritage de l'OUA à l'UA : la quête permanente de l'unité africaine, Kinshasa 2010, cités par BALINGENE KAHOMBO, art. cit., p. 219.

15 Commission du droit international (CDI), 'Rapport de la Commission du droit international - Cinquante-septième session (2 mai-3 juin and 11 juillet-5 août 2005)', Annuaire de la Commission du droit international II (2005), paras. 451-456. 
sations internationales africaines, y compris l'Union des Etats africains (UEA) et l'Organisation de l'unité africaine (OUA), au début des années $1960^{16}$. Un corps de règles régissant les rapports entre les nouveaux Etats indépendants a ainsi commencé à émerger. Déjà, dès la décennie 1960-1970, plusieurs études y ont été consacrées et ont vu dans ces règles, malgré les réticences dues à la novelleté de la matière, l'existence du droit international africain $^{17}$.

C'était, au départ, note encore BALINGENE KAHOMBO, une construction théorique imitative du droit international américain, dont l'existence, communément acceptée, avait été largement évoquée dans l'affaire du Droit d'asile (Colombie/Pérou) devant la Cour internationale de justice (CIJ) en $1950^{18}$. En Afrique, écrit-il, on peut notamment citer les travaux de Boutros Ghali ${ }^{19}$ et de Joseph-Marie BIPOUN-WOUM ${ }^{20}$.

De la sorte, le Professeur Auguste MAMPUYA s'est évertué, en vue de le définir, à inventorier les preuves juridiques de l'existence du droit international africain. Il trouve ces preuves dans la reconnaissance du régionalisme africain, tel que fondé sur le Chapitre VIII de la Charte des Nations Unies, et l'existence d'un système juridique africain ${ }^{21}$. Pour lui ce système constitue un tout contenant plusieurs éléments en interaction et tendant à l'organisation. Il inclue des règles de conduite, des organes d'élaboration et d'application du droit, les sujets de droit (Etats, des institutions de coopération ou d'intégration comme l'UA, les organisations internationales régionales et sous-régionales africaines, les personnes pri-

16 BALINGENE KAHOMBO, art. cit, pp. 218-219.

17 Voir Pierre-François GONIDEC, Note sur le droit des conventions internationales en Afrique, Annuaire français de droit international, XI (1965), 879; François BORELLA, Le régionalisme africain et l'Organisation de l'unité africaine, Annuaire français de droit international, IX (1963), 853. Voir aussi Pierre-François GONIDEC, Existe-t-il un droit international africain?, African Journal of International and Comparative Law, 5 (2) (1993), 243-259; Towards a 'treatise' of African international law', African Journal of International and Comparative Law, 9 (1997), 807-821; TASLIM OLAWALE Elias, Africa and the Development of International Law, Leiden 1972; James T. GATHII, Chapter 17: Africa, in: Bardo Fassbender et Anne Peters (eds.), The History of International Law, Oxford 2012, 407-428.

18 Affaire colombo-péruvienne relative au droit d'asile, Arrêt du 20 novembre 1950: C. I. J. Recueil 1950, 277-278. Voir aussi Opinion dissidente du juge Alvarez, Affaire colombo-péruvienne relative au droit d'asile, 293. D'après le juge Alvarez, 'cette expression 'droit international américain' a été prise dans diverses acceptions qu'il n'y a pas lieu d'indiquer ici. Par ces termes, il faut entendre non pas, comme on pourrait le croire à première vue et comme l'ont cru bien des personnes, un droit international spécial au Nouveau Monde et entièrement distinct du droit international universel, mais l'ensemble des principes, conventions, coutumes, pratiques, institutions et doctrines qui sont propres aux Républiques du Nouveau Monde. Certains juristes veulent appeler cet ensemble 'particularités du droit international en Amérique' : ce n'est qu'une question de terminologie. La dénomination 'droit international américain' a prévalu".

19 BOUTROS BOUTROS-GHALI, L'Organisation de l'unité africaine, Paris 1969, 5.

20 Joseph-Marie BIPOUN-WOUM, Droit international africain: problèmes généraux, règlement des conflits, Paris 1970.

21 Auguste MAMPUYA KANUNK'A TSHIABO, Emergence des Etats nouveaux et droit international, Kinshasa 1984, pp. 196 et 202. 
vées), les faits et actes juridiques, les conceptions philosophiques, historiques, politiques, juridiques et idéologiques qui en déterminent le dynamisme, la cohérence et le fonctionnement, etc. D'après le professeur Auguste MAMPUYA donc, le droit international africain se définit dès lors comme étant « un corps de règles établies dans la région pour régir les relations réciproques des membres de la région, ou une 'situation' régionale ou, encore, pour reprendre l'expression de l'article 52 de la Charte de l'ONU, une situation qui se prête à une action de caractère régional, africain, 22 .

Analysant cette définition, BALINGENE KAHOMBO estime non sans raison que si elle tient globalement la route, elle pèche cependant par une double imprécision. D'abord, que voudrait dire l'expression 'membres de la région' africaine? En fait, deux personnes privées (particuliers, sociétés ou associations), provenant même des pays différents, peuvent être également considérées comme membres de cette région. Mais, la régulation de leurs rapports, y compris au niveau transnational, ne relève pas automatiquement du droit international africain, en l'absence des règles établies à cet effet par les Etats ou les organisations internationales africaines ${ }^{23}$.

Ensuite, on ne perçoit pas très bien dans cette définition l'idée de domaines d'application du droit international africain. Il y est fait référence à l'article 52 de la Charte de l'ONU ${ }^{24}$. Toutefois, il ajoute qu'il faut noter que cette disposition est déjà dépassée par les événements, les accords et organismes régionaux auxquels elle renvoie ayant été envisagés sous l'angle du maintien de la paix et de la sécurité internationale et qu'aujourd'hui, le régionalisme juridique est globalisant et s'étend à tous les domaines du droit, y compris le droit constitutionnel, les droits de l'homme, le droit pénal et le droit économique ${ }^{25}$.

C'est pourquoi on peut plutôt avancer avec lui que "le droit international africain est l'ensemble des règles juridiques adoptées à l'échelle continentale, régionale, sous-régionale, ou dans tout autre cadre bi ou multilatéral, africains, dans le but de régenter les relations entre Etats africains, en quête des solutions aux problèmes qui leur sont spécifiques dans divers domaines de rapports interafricains ou en coopération avec des sujets non-afri-

23 BALINGENE KAHOMBO, Art. cit., pp. 219 -220.

24 Cet article prévoit : «1. Aucune disposition de la présente Charte ne s'oppose à l'existence d'accords ou d'organismes régionaux destinés à régler les affaires qui, touchant au maintien de la paix et de la sécurité internationales, se prêtent à une action de caractère régional, pourvu que ces accords ou ces organismes et leur activité soient compatibles avec les buts et les principes des Nations Unies. 2. Les Membres des Nations Unies qui concluent ces accords ou constituent ces organismes doivent faire tous leurs efforts pour régler d'une manière pacifique, par le moyen desdits accords ou organismes, les différends d'ordre local, avant de les soumettre au Conseil de sécurité. 3. Le Conseil de sécurité encourage le développement du règlement pacifique des différends d'ordre local par le moyen de ces accords ou de ces organismes régionaux, soit sur l'initiative des Etats intéressés, soit sur renvoi du Conseil de sécurité. 4. Le présent Article n'affecte en rien l'application des Articles 34 et $35 »$.

25 BALINGENE KAHOMBO, Art. cit., pp. 219 -220. 
cains. Il s'agit d'un système normatif, comportant des règles de fond, des normes de procédures et celles relatives aux institutions, chargées de leur élaboration ou application ${ }^{26}$.

Aussi, des auteurs se sont-ils donné la peine d'analyser la question liée à l'existence du droit international africain. De ceux-ci se trouve Joseph-Marie BIPOUN-WOUM ${ }^{27}$ qui s'est fixé la tâche difficile de donner une présentation du droit international africain. L'intitulé de son ouvrage pose évidemment le problème essentiel de savoir s'il existe un droit international africain et, dans l'affirmative, si ce droit international africain diffère sensiblement du droit international général et des autres droits internationaux particuliers fondés sur un régionalisme. L'auteur adopte un plan classique : la première partie de son ouvrage est consacrée aux problèmes de base du droit international africain et la deuxième partie aux règlements des conflits interafricains.

Incontestablement, la première partie est celle qui présente le plus d'intérêt pour nous, notamment dans les développements concernant le sujet du droit international africain et l'objet du droit international africain.

Dans l'étude des fondements du droit international africain, l'auteur fait l'inventaire des principaux éléments qui conduisent à l'existence d'un particularisme africain en matière de droit international. Il envisage successivement les fondements sociaux, les fondements historiques et les fondements économiques.

A cet égard, s'il était indispensable de présenter l'originalité du continent africain, pour ce qui concerne l'élaboration d'un droit international original, sa tentative n'est peut- être pas totalement satisfaisante. En effet, il aborde très rapidement l'essentiel des questions qui se posent tant en matière de structures sociales que de l'évolution historique et de la situation économique des Etats africains. La rapidité de l'analyse le conduit à effleurer beaucoup de ces questions sans marquer d'une manière évidente les rapports qui peuvent exister entre les structures sociales et la forme de civilisation africaine avec la nécessité de l'élaboration d'un droit international particulier. Il aurait sans doute été préférable qu'il n'aborde que certaines questions, notamment celle de la situation économique des Etats africains et leur ancienne dépendance coloniale comme éléments déterminants.

Dans son étude du sujet du droit international africain, l'auteur aborde principalement le problème de l'institution étatique et de la construction nationale. On note de bons développements qui sont consacrés à la notion d'équilibre africain, aussi bien équilibre interne de l'Etat qu'équilibre général du continent, comportant nécessairement une harmonisation des politiques et une intangibilité des frontières. Mais au finish, il est d'avis que l'objet du droit international africain est défini comme consistant principalement dans la volonté de coopération interafricaine.

26 Idem, p. 220.

27 Joseph-Marie BIPOUN-WOUM, Le droit international africain. Problèmes généraux, règlement des conflits, Tome V, Patis, LGDJ, 1970. 


\section{B. Les mécanismes africains de protection des droits de l'homme}

Sur le continent africain, les droits de l'homme bénéficient d'une protection à la fois au niveau régional et au niveau sous-régional principalement à travers l'action des juridictions attachées aux organisations communautaires sous-régionales.

\section{Au niveau régional}

Le point de départ de toute exégèse textuelle des droits de l'homme en Afrique indépendante, pour reprendre l'expression de SAIDOU NOUROU TALL, est, sans aucun doute, la Charte de l'OUA de 1963, qui pose les grands principes et en énonce, de manière fort évasive, quelques règles ${ }^{28}$.

Bien avant la création de l'OUA, en réaction aux crimes et exactions coloniaux, ${ }^{29}$ dans la perspective des indépendances prochaines, les juristes africains réunis en 1961 à Logos avaient tiré la sonnette d'alarme pour une prise en charge des droits de l'homme en Afrique. L'adoption de la charte de l'OUA en 1963 en offrira une faible assise normative.

Les Etats africains se contenteront d'ébaucher des principes dans le préambule de la charte, tels que le droit des peuples à disposer d'eux-mêmes ( $\$ 1)$, de même que l'idée selon laquelle « la liberté, l'égalité, la justice et la dignité sont des objectifs essentiels à la réalisation des aspirations légitimes des peuples africains » $(\S 2)$; la libre disposition des ressources naturelles et humaines $(\S 3)$; la coopération et la solidarité $(\S 4)$; la paix et la sécurité (§5); la nécessité de la sauvegarde et de la consolidation « de l'indépendance et de la souveraineté durement conquises ainsi que l'intégrité territoriale de nos Etats et à combattre le néocolonialisme sous toutes ses formes » (\$6).

Les Etats africains proclamaient aussi leur adhésion à la Charte des Nations Unies et à la Déclaration Universelle des Droits de l'Homme et leur idéal de réalisation de l'unité du continent.

En dehors du préambule, le corpus juris de la Charte de l'OUA contient quelques considérations générales sur les droits de l'homme et les libertés fondamentales. On peut s'en convaincre à la lecture des dispositions sur les objectifs de l'OUA (Art. II), les principes axiologiques de la charte (Art. II), le principe pacta sunt servanda (Art. VI); le règlement des différends avec la Commission de Médiation et de Conciliation Africaine (Art. XIX).

Au total, la Charte de l'OUA ne reflétait qu'une approche souverainiste des Etats africains qui, à l'épreuve, n'avaient d'autres soucis que de conforter leurs indépendances récentes, englués qu'ils étaient dans des querelles des leadership et d'idéologie (pro-capitaliste, pro-communiste, voire de non alignement), et peu enclins à ouvrir la boite de pandore

28 41. SAIDOU NOUROU TALL, Droit du contentieux international africain, Dakar, L'HarmattanSénégal, 2018, p. 281.

29 L.S. SENGHORE en 1950 proposait que la Convention Européenne des droits de l'homme s'appliquant aux territoires colonisés par les Etats parties. 
des revendications individuelles des droits de l'homme, l'unité nationale et la prédominance des partis uniques étant alors leurs baromètres communs ${ }^{30}$.

Cette situation va perdurer jusqu'au tournant des années 80 marquées par l'avènement de la charte africaine des droit de l'homme et des peuples de $1981,{ }^{31}$ qui créait la commission africaine de Banjul.

Par ailleurs, le contexte de la fin de la guerre froide, l'avènement des conférences nationales souveraines des années 1990, la conversion aux vertus du multipartisme, de la démocratie et de la bonne gouvernance, souvent sous l'impulsion de l'ONU ou des institutions de Bretton Woods, enfanteront un environnement encore plus propice à l'énoncé et aux garanties de droits. Dans ces circonstances, l'OUA ne pouvait en remplaçant l'OUA, qu'adapter les anciens textes et adopter les nouvelles conventions.

En tant qu'organisation qui succède à l'OUA, elle va reprendre tous les textes des droits de l'homme adoptés avant 2000 tout en y adjoignant des dispositions résultant des nouvelles avancées structurelles et normatives. Et pour marquer l'évolution de cette promotion et de cette protection de droits de l'homme, il sied d'emblée de revenir sur l'articulation entre la commission et la cour Afrique des droits l'homme et des peuples, avant d'en évoquer les prolongements et avatars qui s'en ont suivis même au niveau sous-régional africain.

Nous nous limiterons ainsi dans ce point à examiner le système non juridictionnel de sauvegarde et de protection des droits de l'homme notamment à travers la Commission africaine des droits de l'homme et des peuples. Bien entendu, à la lecture de la Charte africaine des droits et du bien-être de l'enfant, il y a lieu d'ajouter un autre mécanisme dénommé Comité africain d'experts sur les droits et le bien-être de l'enfant ${ }^{32}$. Mais celui-ci ne sera pas abordé ici alors que le mécanisme juridictionnel fera l'objet d'étude du point 3 .

Ceci étant, il importe de relever avec KANDOLO ON'UFUKU wa KANDOLO, P.-F. que la consécration d'un système africain de protection des droits de l'homme est le résultat d'un long processus qui est d'ailleurs encore en cours de maturation. Parmi les facteurs explicatifs (mais non justificatifs) de cette lenteur, le Doyen BABACAR KANTE cite les conséquences du parti unique, des conflits, de la dégradation de l'environnement, de la pauvreté, du VIH/SIDA et plus récemment du terrorisme ${ }^{33}$.

Ainsi, si l'idée de la création d'une Commission africaine chargée de promouvoir et de protéger les droits de l'homme est relativement ancienne, car née immédiatement après les indépendances, elle n'a été concrétisée qu'avec l'adoption, en 1981, de la Charte africaine des droits de l'homme et des peuples. Mais avant cette date, le corpus des règles issues des

30 SAIDOU NOUROU TALL, Op. cit., p. 282.

31 Adoptée à Nairobi le 27 Juin 1981 et entrée en vigueur le 21 Octobre 1986.

32 KANDOLO ON'UFUKU wa KANDOLO, P.-F., Du système congolais de promotion et de protection des droits de l'homme. Contribution pour une mise en cuvre du mécanisme institutionnel spécialisé, Mémoire de D.E.A, Faculté de Droit, UNILU, 2011, p. 174.

33 Idem, pp. 174 et suiv. 
Conventions internationales initiées par l'ONU constituait les seules références communes des pays africains ${ }^{34}$.

C'est en effet avec cette Charte que les Etats africains ont progressivement élaboré et adopté, en tant que manifestation aussi du régionalisme juridique africain, des normes spécifiques à leur continent et mis en place des mécanismes tendant à les garantir.

Il s'agit d'un système, écrit l'auteur précité, citant BABACAR KANTE, dans la mesure où il constitue « un ensemble cohérent d'éléments destinés à poursuivre un même objectif, en l'occurrence la promotion et la protection des droits de l'homme en Afrique $»^{35}$.

Et de fait, la Charte africaine des droits de l'homme et des peuples prévoit, dans sa dixième partie, des mesures de sauvegarde des droits de l'homme. Ainsi, son article 30 dispose : «Il est créé auprès de l'Organisation de l'Unité Africaine une Commission Africaine des Droits de l'Homme et des Peuples(...) chargée de promouvoir les droits de l'homme et des peuples et d'assurer leur protection en Afrique ».

Pendant longtemps, cette Commission est restée la seule institution continentale chargée de veiller au respect des droits de l'homme en Afrique.

Créée en même temps que l'adoption de la Charte, la Commission africaine des droits de l'homme et des peuples avait été, en 1981, conçue comme un mécanisme de promotion et de protection des droits de l'homme en Afrique. L'article 45 de la Charte révèle, en effet, que la mission de promotion était beaucoup plus prépondérante que celle de protection, affirmée à peine avec retenue et circonspection. La Commission est entrée en fonction depuis le 2 novembre 1987. Elle siège à Banjul (Gambie) et se réunit en session ordinaire deux fois par an, aux mois de mai et novembre ${ }^{36}$.

C'est le 13 février 1988 que la Commission a adopté son Règlement intérieur ${ }^{37}$.

S'agissant de ses attributions et procédures, la lecture de l'article 45 de la Charte africaine des droits de l'homme et des peuples, donne à comprendre que la Commission exerce les fonctions de promotion et de protection des droits de l'homme, d'interprétation de la Charte et d'exécution des tâches qui lui seront confiées par la Conférence des chefs d'Etat et de gouvernement.

En ce qui concerne la protection des droits, ses compétences consistent à recevoir des communication-négociations ${ }^{38}$, des communications-plaintes ${ }^{39}$, des communications autres que celles émanant des Etats parties ${ }^{40}$.

34 Ibidem.

35 BABACAR KANTE, « Le système africain de protection des droits de l'homme " in INSTITUT INTERNATIONAL DES DROITS DE L'HOMME, Op. cit, pp. 40-55, cité par KANDOLO ON'UFUKU wa KANDOLO, P.-F., Op. cit., p. 175.

36 KANDOLO ON'UFUKU wa KANDOLO, P.-F., p. 176.

37 Ibidem.

38 Voir les articles 47 de la Charte et 87 à 91 du Règlement intérieur de la Commission africaine des droits de l'homme et des peuples.

39 Voir les articles 48 et 49 de la Charte et 92 à 100 du Règlement intérieur de la Commission.

40 Article 55 de la Charte. 
Quant à la compétence personnelle, devant la Commission, les Etats, mais aussi les individus ou les organisations non gouvernementales ont qualité à agir. Et lorsqu'elle est saisie, la Commission peut, en application de l'article 46 de la Charte, recourir à « toute méthode d'investigation appropriée ».

Avec Jean-Louis ATANGANA AMOUGOU, on peut noter à propos de la saisine de la Commission par les individus que « (...) la Charte africaine ne mentionne pas expressément les requêtes individuelles. Son article 55 se contente d'énoncer qu' " avant chaque session, le Secrétaire de la Commission dresse la liste des communications autres que celles des Etats parties à la Charte et les communique aux membres de la Commission qui peuvent demander à en prendre connaissance et en saisir la Commission ».

Mais en vertu de sa compétence d'interprétation, la Commission avait alors procédé à une lecture extensive de cette disposition. Cette lecture généreuse de la Charte lui avait déjà permis de recevoir et de traiter les requêtes individuelles dont l'examen n'était pas expressément prévu par la Charte. Elle a examiné à ce jour plus de 250 communications dont la quasi-totalité porte sur les requêtes individuelles au titre de l'article 55 de la Charte. Pour pouvoir remplir convenablement ses missions, la Commission avait ainsi dû faire preuve d'une certaine audace et sortir du champ étroit de son action dans lequel la Charte l'avait confiné. Elle avait ainsi bravé l'obstacle de la confidentialité de ses activités en procédant à une interprétation extensive de la Charte $»^{41}$.

Néanmoins, certains critiques ne se sont pas empêchés de relever ce qu'ils considèrent comme étant la défaillance de la Commission africaine laquelle a, de leur avis, nécessité la naissance de la Cour Africaine des droits de l'homme et des peuples ${ }^{42}$.

C'est que ces auteurs indiquent que le bilan de la Commission africaine est mitigé, et la principale raison tient à sa nature même ${ }^{43}$. Selon eux, le système mis en place au sein de la Commission avait d'ailleurs été critiqué, à tort ou à raison, par presque tous les commentateurs. Du fait de son caractère non juridictionnel, la Commission n'avait pas de moyens de contrainte sur les Etats parties. En outre, ses rapports étaient soumis à la Conférence des Chefs d'Etat et de gouvernement, un organe politique qui, seul, avait le pouvoir d'autoriser leur publication. De même le suivi de la mise en œuvre ou de l'exécution des mesures n'était pas garanti. Les Etats ont d'ailleurs rarement utilisé leur droit de saisine de la Commission ${ }^{44}$.

Il en va d'autant plus ainsi que par exemple la Commission a curieusement été muette sur des questions d'une particulière gravité comme le génocide du Rwanda, les crimes com-

41 J-L. ATANGANA AMOUGOU, « Avancées et limites du système africain de protection des droits de l'homme : la naissance de la Cour africaine des droits de l'homme et des peuples " in Droits fondamentaux, $\mathrm{n}^{\circ} 3$, janvier-décembre 2003 [en ligne], décembre 2003, <www.droitsfondamentau x.org> (page consultée le 30 juin 2010), cité par KANDOLO ON'UFUKU wa KANDOLO, P.-F., p. 177.

42 Idem.

43 Lire par ex. BABACAR KANTE, Op. cit., p. 46.

44 KANDOLO ON'UFUKU wa KANDOLO, P.-F., pp. 177 et suiv.. 
mis par des troupes dites d'agression lors de la guerre en République Démocratique du Congo. Et les affaires sur lesquelles la Commission s'est prononcée clairement sont essentiellement le fait des organisations non gouvernementales. Elle ne peut néanmoins pas connaître d'une affaire qui lui est soumise qu'après s'être assurée que tous les recours interne, s'ils existent, ont été épuisés, à moins qu'il ne soit manifeste pour la Commission que la procédure de ces recours se prolonge d'une manière anormale ${ }^{45}$.

Mais malgré tout cela, faut-il le saluer, la Commission a contribué d'une certaine mesure au développement des droits de l'homme en Afrique. Elle a en effet dégagé un certain nombre de principes et directives portant sur des droits et libertés fondamentaux : liberté d'expression, les traitements inhumains, le procès équitable et l'assistance légale.

En effet comme le renseigne si pertinemment bien SAIDOU NOUROU TALL ${ }^{46}$, et si la préférence envers une véritable cour plutôt qu'en une commission des droits de l'homme est bien connue, cela n'a pas empêché de saluer l'œuvre de la comm.ADHP. Celle-ci a fourni un rapport non négligeable ${ }^{47}$ dans la densification de la protection et de la promotion des droits de l'homme.

Faisant preuve d'audace, la commission a, de l'avis de l'auteur précité, manié avec dextérité les principes d'interprétation téléologique et raisonnablement extensive pour offrir un contenu concret aux droits et protections des individus et des groupes.

Aussi, dans l'Afrique Monim Elgak, Osman Hummeida et Amir Suleiman (représentés par FIDH et Organisation Mondiale contre la Torture) en République du Soudan, elle consacre la nécessité du respect du droit à l'information, du droit à la Santé, du droit du travail $^{48}$.

La commission s'est, à maintes reprises, intéressée aux divers cas d'arrestations et de tortures d'officiers suspectés de tentatives de coups d'Etat ${ }^{49}$, aux problèmes constitutionnels relatifs à la nationalité ${ }^{50}$ ou aux élections législatives, ${ }^{51}$ à la promotion et au respect des

45 Article 50 de la Charte.

46 SAIDOU NOUROU TALL, Op. cit., p. 291 et suiv..

47 Elle a reçu plus de 400 communications et a rendu soit des décisions d'irrecevabilité, soit des décisions d'incompétence, soit des décisions sur le fond déboutant les plaignants ou reconnaissant l'allégation de la violation des droits.

48 Communication n³7/09, Affaire Monium Fgak. Osman Hummedia et Amir Sukiman (représenté par FIDH et Organisation mondiale contre la torture) en République du Soudan.deciston du 14 mars 2014.

49 Communication $n^{\circ} 27 / 03$ et 28/03, Affaire luterights. ASADHO et Maitre () Disc/ République Démocratique du Congo, Novembre 2013.

50 Communication n³18/06, Affaire Open Society Justice initiative et République de Cote d'Ivoire, décision de février 2015.

51 Communication ${ }^{\circ} 433 / 12$, Affaire Albert Biallufu Ngandu et République Démocratique du Congo, décision du 25 février 2016. 
droits de l'enfant, ${ }^{52}$ à la conciliation entre droit de propreté et expropriations, ${ }^{53}$ ou encore les récurrents détournements de derniers publics, et le problème de refus d'agrément d'ONG ${ }^{54}$

Beaucoup d'autres affaires méritent de retenir l'attention. Il en est ainsi, de l'Affaire Gaétan Bwampamye (Avocats sans frontières c. Burundi), elle a estimé que " le Burundi a ignoré les engagements des cours tribunaux à se conformer aux normes internationales en vue d'assurer un procès équitable »; comme de l'affaire Legal Ressources Foundation vs. Zambia et celle des militaires nigérians condamnés à mort par le régime du Général Sani ABACHA (Commission 28/8, § 4).

Dans l'affaire dite Ken Saro-Wiwa, la commission s'érige en gendarme de sa mission « de l'obligation positive de prévenir la violation des droits de l'homme » du fait que « la charte Africaine a été élaborée et a reçu l'adhésion volontaire des Etats Africains désireux d'assurer le respect des droits de l'homme sur ce continent. Une fois ratifiée, les Etats parties à la charte sont légalement liés par ses dispositions. Un Etat qui ne veut pas respecter la charte Africaine aurait dû ne pas la ratifier. Une fois légalement lié cependant, l'Etat doit respecter la loi au même titre que l'individu $»^{55}$.

La comm.ADHP a eu à traiter des droits collectifs notamment des droits fondamentaux de peuples autochtones comme les Vhavenda ${ }^{56}$. Elle a consacré la notion de «violation continue » pour connaitre des faits survenus avant l'entrée en vigueur de la CADHP, mais qui perdurent près cette date ${ }^{57}$ sous la réserve qu'en principe l'Etat ne saurait être tenu responsable d'événement ${ }^{58}$ avant que la charte ne lui soit applicable.

De même, la commission estime que la violation, prima facie, d'un droit garanti par la CADHP doit être suffisamment démontrée pour qu'une affaire soit recevable. ${ }^{59}$ Elle

52 Communication $\mathrm{n}^{\circ} 325 / 06$ Affaire Organisation mondiale contre la torture et ligue de la zone Africaine pour la défense des droits des enfants et élèves (pour les couples de Celine). En République Démocratique du Congo décision du 18 décembre 2015.

53 Communication n³89/10 Affaire Mhidmbak Geneviève et République du Cameroun décision du 06 mai 2015.

54 Communication n ${ }^{\circ} 416 / 12$ Affaire Jean-Maurice Atangana Mebara et République du Cameroun, décision du 7 Aout 2015.

55 Communication $n^{\circ} 154 / 96$. $§ 43,48$, cité par Roger Koussetogue KOUDE, « les droits de l'homme : de l'inituition universaliste à l'universalité récusée ». Révue Trimestrielle des dcroits de l'homme n $68 / 2006$, pp. 909-938.

56 Communication $n^{\circ} 335 / 06$, Affaire Dobalorivhuwa patriotic Front et République d'Afrique du Sud, décision du 23 Avril 2013.

57 Annesty International of Soudan, Communication n ${ }^{\circ} 48 / 90, n^{\circ} 52 / 90$ et n89/90. Annette Pagnoule (au nom d'Abdoulaye Mazou) c/ Cameroun communication 39/90.

58 Huitième Rapport d'Activités, ibid, Annexe VI.

59 Ligue camerounaise des droits de l'homme et Cameroun; Frederick Korvah c/Liberia, Communications n ${ }^{\circ} 69 / 96$ et $n^{\circ} 1 / 88$; Affaire Griebenow (au nom de David Ashley Price) c/Afrique du Sud, Communication $n^{\circ} 315 / 06$. 
contrôle l'application des droits des réfugiés. ${ }^{60}$ Elle a défini la notion de discrimination de l'article 2 de la CADHP comme « tout acte qui vise la distinction, l'exclusion, la restriction ou la préférence fondée sur des motifs tels que la race, la couleur, le sexe, la langue, la religion, l'opinion politique ou autre l'origine nationale ou sociale, la fortune, la naissance ou toute autre situation et qui a pour objet de dernier ou compromettre la reconnaissance, la jouissance ou l'exercice par tous, dans des conditions d'égalité, de tous les droits et libertés $»^{61}$.

Elle identifie trois critères de détermination de la discrimination : «si des cas pareils sont traités de manière différente; si une différence de traitement n'a pas de justification objective et raisonnable et, s'il n'y a pas de proportionnalité entre le but visé et les moyens employés $»^{62}$.

Elle n'hésite pas à rappeler l'Affaire Brown vs/Board of Education of Topeka de la Cour Suprême des Etats-Unis (1954) en traitant du droit à une égale protection de la $1 \mathrm{loi}^{63}$ ni les observations générales du Comité des droits de l'homme de l'ONU ${ }^{64}$ sur le droit de participation aux affaires publiques et l'accès aux services et biens publics, selon l'art 13 de la CADHP.

Elle a précisé les droits sociaux dans beaucoup de ses décisions, notamment le droit de travailler dans des conditions équitables, ${ }^{65}$ le droit à travail égal salaire égal en se référant à l'arrêt Etim Moses de la cour d'Abuja ${ }^{66}$ et à l'observation générale $n^{\circ} 18$ du Comité sur les droits sociaux, économiques et culturels ${ }^{67}$.

Elle a traité du droit à la libre association ${ }^{68}$ des effets des coups d'Etat (restriction des droits et libertés, restauration de la peine de mort, violations diverses) dans l'affaire sir Dawda K. Jawara c. Gambie (communication 14/ 95) ${ }^{69}$, des atrocités commises au Darfour,

60 Mouvement des réfugiés mauritaniens au Sénégal c/Etat du Sénégal, communication n ${ }^{\circ} 162 / 97$.

61 Affaire Zimbabwe lawyers for Human Rights et Institut pour les droits humains et le développement en Afrique (au nom de Andrew Barclay Meldrum), communication n²93/04, décision de 2006.

62 Affaire Kenneth Good c/Botswana (2010), communication 315/05.

63 Dans l'affaire institut pour les droits de l'homme et le développement en Afrique (pour le compte de Esmaila Connateh et 13 autres) c/Angola (2008).

64 Voir le rappel de l'observation générale n²5 du comité, dans l'Affaire Dabalorivhuwa, précitée, $\S 128$.

65 Affaire Zimbabwe Lawyers for Human Rights. Associated Newspapers of Zimbabwe c/ Zimbabwe, communication $n^{\circ} 284 / 03$ (2008): voir aussi communications $n^{\circ} 292 / 04$ (2008) et $n$ ๑39/90 (1997) précitées.

66 Affaire Etim Moses c/Gambie (2007) de la cour de justice CEDEAO.

67 Sur « le droit au travail », comité sur les droits économiques, sociaux et culturels (2003).

68 Dans l'affaire civil liberties organiastion (au nom du Nigeria Bar Association c/Nigeria (communication $\left.\mathrm{n}^{\circ} 101 / 93\right)$.

69 Sur la commission, en général, consulter Evelyu A. ANKULUMANI, The African commission on Human and people's Rights : practice anti productures, Dordrecht, Martinius Nijhoff publishers, 1996. 
expulsion forcées et autres violations du DIH et reconnait le droit à l'eau et le droit des peuples aux droits économiques, sociaux et culturels (Affaire Centre on Housing Rights and Evictions(COHRE) c/Soudan $)^{70}$.

Par ailleurs, elle a rendu un avis juridique, suite à l'adoption, le 29 Juin 2006 par le Conseil des droits de l'homme, de la Déclaration des Nations-Unis sur les droits des peuples autochtones ${ }^{71}$.

Elle a dressé une véritable ligne directrice jurisprudentielle sur l'épanouissement des voies de recours, en estimant qu' « il est serait incorrect d'obliger les plaignants à user des voies de recours qui ne fonctionnent pas de façon impartiale et qui ne sont pas tenues de statuer conformément aux principes de droit. Le recours n'est ni adéquat ni efficace $»,{ }^{72}$ qu'on ne saurait exiger l'épanouissement si « des voies de recours internes (...) ne sont ni disponibles ni pratiques $»^{73}$. Dans ce sillage, la Comm.ADHP, dans l'affaire précitée Ken Saro-Wiwa, retient que le « fait de harceler les avocats de la partie civile, de les menacer dans leur vie privée et/ou professionnelle jusqu'à les pousser à renoncer aux poursuites engagées... prive les plaignants d'une bonne administration de la justice et de la possibilité d'épuiser les voies de recours internes ${ }^{74}$.

La commission, en tant que mécanisme de contrôle de l'application de la CADJP avec son double mandat de promotion et de protection des droits de l'homme, a eu l'occasion de statuer sur la détention arbitraire et les traitements inhumains et dégradants ${ }^{75}$, sur le droit à un procès équitable ${ }^{76}$, sur les libertés d'expression et d'association ${ }^{77}$, la discrimination et

70 Communication 296/2005, décision du 29 Juillet 2009. La commission reconnait le droit à l'eau comme implicite dans la charte; se fonde sur l'art.22 pour reconnaitre les DESC du peuple darfourien; s'intéresse aux violations du droit à la vie ou à une vie libre de torture, aux déplacements forcés, au droit à l'intégrité physique (art. 4 CAPDHP) au droit au meilleur état de santé que la personne est capable d'atteindre (art. 16).

71 www.achpr.org.site de la commission.

72 Affaire constitutional rights project c/Nigeria, communication 60/91.

73 Affaire RADDHO et Zambie communication 71/92; Affaire relative aux expulsions des Burindais, du Rwanda en 1989, communication portant $n^{\circ} 27 / 89 n^{\circ} 49 / 91$ et $n^{\circ} 99 / 93$ : Affaire Forum of conscience c/Sierra Leonne. Communication n ${ }^{\circ} 223 / 98 \S 15$.

74 Voir Roger Koussetogue KOUDE, « les résistances à l'universalité des droits de l'homme au titre de la pensée politique », en Denis MAUGENEST et Théodore THOLO. Op. cit, pp. 109 421, SaidouNourou TALL, «La problématique du respect des droits de l'homme en période de conflit armée », in Denis MAUGINESE et Théodore HOLO, op.cit.pp.65 77.

75 Affaire John D.Ouko c/Kenya, communication n²32/99. Affaire curtis Francis Doebbler c/ Soucken. Communication n²32/2000 : Affaire Haregenewain Gabré Sélassié et IHRDA (au nom des fonctionnaires de l'époque Dergue)c/ République Féderale et Démocratique d'Ethiopie, communication n³01/2005, décision de 2013.

76 Affaire Law Office of Glurel Souleiman c/Soudan, communication n²22/98 et n²99/99.

77 L'affaire civil libertés organisation, c/Nigeria,précitée. 
les expulsions d'étrangers ${ }^{78}$, sur l'environnement et la santé ${ }^{79}$, sur les violations commise en temps de guerre par des parties privées ${ }^{80}$.

Elle s'est prononcée sur les conditions de détention et de traitement des malades mentaux ${ }^{81}$; sur l'inefficacité et l'inaccessibilité de recours internes pour un évadé 82 , pour un Etudiant $^{83}$; sur la prolongation anormale de procédures internes ${ }^{84}$; la recevabilité de la plainte même si la situation s'est améliorée après le dépôt de la communication ${ }^{85}$; sur la recevabilité même en cas de médiation en cours par des OIG comme l'OUA ou L'UE ${ }^{86}$, sur la non exclusion du règlement à l'amiable ${ }^{87}$, et sur la possibilité des réparations ou autres mesures appropriées ${ }^{88}$.

Sur le thème des réparations, l'affaire Institute for Human Rights and Developpement in Africa, Rights and Accountability in developpement et Action contre l'impunité pour les droits Humains, c/République du Congo, décision du 4 Aout 2017, est l'une des plus édifiantes en ce que la commission établit la responsabilité de la RDC, dans le massacre de plus 70 personnes à Kilwa et la condamne à verser une indemnité de 2,5 millions de dollars USD à leurs familles et autres victimes, tout en réprimandant la société minière australocanadienne Anvil Mining pour avoir aidé au transport et soutenu logistiquement les soldats de l'armée congolaise. La commission exige une nouvelle enquête criminelle, la présentation par l'Etat d'excuses aux habitants de Kilwa, l'exhumation et la réinhumation des corps jetés dans une fosse commune, la construction d'un mémorial, la reconstruction de l'école et de l'hôpital et la fourniture d'une assistance psycho-sociale aux personnes affectées, sans ignorer de lui rapporter dans les 180 jours la suite réservée à ses recommandations ${ }^{89}$.

78 Affaire Fedération internationale des ligues des droits de l'homme.

Union interafricaine des droits de l'homme, RADDHO, ONDH au Sénégal et association Malienne des droits de l'homme c/Angola, communication n ${ }^{\circ} 159 / 96$.

79 Affaire social and Economic Rights Action center (SERAC) c/Nigeria, communication n $155 / 96$.

80 Affaire commission nationale des droits de l'homme et des libertés, c/Tchad, communication $74 / 92$.

81 Affaire purohit et Moore c/Gambie, communication 24/01.

82 Affaire Alhassan Abubakar, c/Ghana, communication 103/93.

83 Affaire Rights international c/Nigeria, communication 215/98.

84 Affaire Kenya Human Rights Commission c/Kenya, communication n $135 / 94$.

85 Communication $n^{\circ} 68 / 92$ et nº $78 / 92$ (respectivement Krishna Achutan et Malawi et Amnesty international c/Malawi.

86 Affaire Embgba Mekongo Louis c/Cameroun, communication, $\mathrm{n}^{\circ} 59 / 91$.

87 Art. 52 charte 1981 et communication $n^{\circ} 25 / 89$, $n^{\circ} 47 / 90, n^{\circ} 56 / 91$ et $N^{\circ} 100 / 93$. Free Legal Assistance Group lawyers commitee for HumanRights. Union interafricaine des droits de l'homme. Les témoins de Jehova c/Zaïre.

88 Art. 27, protocole de 1998 et communications $n^{\circ} 54 / 91, n^{\circ} 61 / 91, n^{\circ} 98 / 93, n^{\circ} 154 / 97$ et $n^{\circ} 210 / 98$. Amnesty international, Ms. Sarr Diop, UIDII and RADDIIO. Collectif des veuves et ayants-droits. Association Mauritanienne de droit de l'homme c/Mauritanie.

89 SAIDOU NOUROU TALL, Op. cit., pp.297-298. 
Malgré cette impressionnante somme « jurisprudentielle », l'absence du caractère obligatoire (conditionnée à l'acceptation de la Conférence des Chefs d'Etat et de Gouvernement) de ses décisions, combinée à la confidentialité (certes de plus en plus réduite, mais encore résiduelle) de certaines procédures, ont amené le double constat : d'une part, d'un déficit profond dans l'accomplissement de la mission de sauvegarde des droits de l'homme et des peuples et, d'autre part, de la nécessité de la compléter, ainsi que nous le verrons par ailleurs, par l'adjonction d'une Cour des droits de l'homme. Encore est-il à considérer que l'existence même de la Commission Africaine des Droits de l'homme et des Peuples a constitué un puissant aiguillon non seulement pour la mise en place d'une Cour Africaine des Droits de l'Homme et des Peuples ${ }^{90}$, mais aussi pour la prise en charge desdits dits par les organisations sous-régionales africaines.

\section{Au niveau sous-régional}

L'espace juridictionnel africain est actuellement parsemé de plus d'une demi-douzaine de juridictions actives à caractère régional ou sous-régional. Il s'agit pour ces dernières, des mécanismes mis en place par les Organisations internationales, essentiellement économiques mais parfois juridiques, des Etats de l'Afrique l'Organisation pour l'Harmonisation du Droit des Affaires en Afrique (O.HA.D.A.), la Communauté Economique des Etats de l'Afrique de l'Ouest (C.E.D.E.A.O.), la Communauté Économique des Etats de l'Afrique Centrale (C.E.E.A.C.), la Communauté de Développement de l'Afrique Australe (S.A.D.C.), la Communauté des Etats de l'Afrique de l'Est (C.E.A.E.), etc.

Ces organisations ont pour vocation initiale, on le sait, de promouvoir l'intégration économique et/ou juridique de leurs Etats membres respectifs. Elles ont été toutefois amenées progressivement à élargir leurs compétences, par voie statutaire ou de manière pragmatique, à des activités et programmes relevant des domaines des normes conventionnelles de droits de l'homme, du maintien de la paix et de la sécurité internationales ainsi que du désarmement $^{91}$.

C'est que depuis les années 80 , un formidable mouvement en faveur de la protection et de la promotion des droit de l'homme n'a pas seulement été observé au niveau de l'OUA/UA mais également amené les autres organisations internationales africaines à s'intéresser de manière incidenté aux droits de l'homme, alors même que leur vocation première, nous le savons, était plutôt orientée vers des thématiques plus générales comme le développement économique, l'intégration ou la coopération politique. C'est pourtant la CEDEAO qui a eu le mérite d'étoffer, le plus, sa production normative dans ce domaine.

90 Idem, p. 298.

91 Lire à ce propos MUTOY MUBIALA, Le système régional africain de protection des droits de l'homme, coll. Organisation internationale et relations internationales, $\mathrm{n}^{0} 59$, Bruxelles, Bruylant, 2005. 
D'autres organisations sous régionales africaines sectorielles ont également accompagné le mouvement vers la démocratie et le respect des droits de l'homme ${ }^{92}$.

Ainsi la quasi-totalité des organisations régionales et sous-régionales africaines sont des organisations d'intégration économique hormis l'Organisation pour l'Harmonisation en Afrique du Droit des Affaires qui, elle, constitue une organisation d'intégration juridique. Il s'agit en effet d'une organisation internationale créée à la suite du Traité relatif à l'harmonisation du droit des affaires en Afrique signé le 17 octobre 1993 à Port-Louis et révisé à Québec, au Canada, le 17 octobre 2008 dans l'objectif d'améliorer la sécurité juridique et judiciaire en Afrique par le biais du développement de l'intégration juridique des Etats signataires.

De la sorte Jean Joss MILINGO ELLONG suggère qu'en droit de l'OHADA et de l'Union africaine, les droits fondamentaux soulève pourtant d'autres questions qui devraient sans doute retenir notre attention. Peut-on par exemple véritablement parler de droits fondamentaux en droit de l'OHADA ou de l'Union africaine? Existe-t-il dans ces espaces juridiques des mécanismes juridictionnels de contrôle et de sanction de la violation des droits fondamentaux?

L'auteur se rend à l'évidence le fait que si les droits fondamentaux relèvent du champ de compétence de l'Union africaine, à travers principalement la Charte africaine des droits de l'homme et des peuples ${ }^{93}$, tél n'est pas forcément le cas en droit de l'OHADA. Pour certains auteurs d'ailleurs, le droit de l'OHADA est indifférent aux droits fondamentaux parce que ce dernier n'aurait vocation qu'a connaitre des problématiques du commerce et des activités économiques, ou que « les actes uniformes subséquents ne font [pas] expressément référence aux droits de l'homme [ou fondamentaux] $»^{94}$.

A partir d'une telle affirmation, l'on peut se demander avec lin Jean Joss MILINGO ELLONG, si le fait pour un texte ou pour des décisions de ne pas expressément mentionner un droit ou un principe comme fondamental suffit dès lors à nier son existence? Si tel était le cas, le principe de l'autonomie de la volonté connu en droit des obligations n'aurait pas forcément eu de beaux jours devant lui, et ne serait actuellement qu'une peau de chagrin ${ }^{95}$.

C'est que le droit de l'OHADA est, à l'instar du droit de l'Union africaine, un berceau de droits fondamentaux de la personne. Plus exactement, le droit de l'OHADA est l'application sectorielle des droits fondamentaux reconnus par l'Union africaine. Le respect de ces droits peut parfois être source de tension. A titre d'exemple, le droit à l'égalité manifeste dans certains Actes uniformes, et plus spécialement dans l'Acte uniforme relatif au droit commercial général et aux sociétés commerciales, est une application concrète des prin-

93 Voir le Préambule de la Charte africaine des droits de l'homme et des peuples.

94 Lire Jean Joss MILINGO ÉLLONG, Les conflits de normes en matie re de droits fondamentaux : le cas de l'OHADA et de l'Union africaine, in, Bulletin de droit économique, Université Laval, (2014) 2 B.D.E., pp. 28. 
cipes d'égalité, de l'égalité devant la loi et du droit de participation à la direction des affaires publiques du pays posés par la CADHP. En outre, la liberté entrepreneuriale posée par ces mêmes textes est la résultante de la liberté professionnelle, de la liberté d'association, de la liberté de circulation et de la liberté d'établissement, toutes prévues par la CADHP. Par ailleurs, l'Acte uniforme sur les sûretés contient un faisceau de règles relatives au droit de propriété énoncé par la CADHP. Enfin, pour ne citer que ces quelques exemples, les « projets et avant-projets d'Acte uniforme en cours» ${ }^{96}$ sont le réservoir de droits fondamentaux reconnus simultanément par la CADHP et l'OHADA. Il s'agit notamment du droit de la personne au respect de sa dignité et de sa vie privée et familiale sous toutes ses formes ${ }^{97}$.

La protection juridictionnelle des droits fondamentaux quant à elle est assurée par plusieurs juridictions. En droit de l'OHADA par exemple, la Cour commune de justice et d'arbitrage (CCJA) est, nous dit-on, « gardienne de la bonne application du droit uniforme et de la célérité du procès $»^{98}$; y compris le juge national, entendu comme le juge régional de droit commun.

Somme toute, il faut donc le constater pour s'en réjouir, les droits fondamentaux ne sont pas l'apanage du seul droit de l'Union africaine. Ils sont également pris en compte par l'architecte régional de l'OHADA. La sanction de leur inobservation peut être prononcée par les juridictions de ces deux organisations tout comme par celles d'autres juridictions sous-régionales.

Dans ce sens, le Tribunal de la SADC, dont les activités sont depuis suspendues, attend toujours l'adoption de la Charte devant élargir sa compétence pour connaitre des litiges relatifs aux droits de l'homme. Pourtant, il a déjà eu par le passé à se prononcer sur les droits de l'homme dans quelques affaires dont celle opposant certains ressortissants zimbabwéens au Zimbabwe : c'est l'affaire Fick and Fours others v Zimbabwe (Case SADC(T) 01/2010).

Quant à la Cour de justice de la CEAE, elle ne dispose pas d'un mandat aussi clair que celui de la Cour de la CEDEAO qui, elle, en matière des droits de l'homme, dispose d'un mandat clair. Ainsi aux termes de l'article 4 du Traité de la CEDEAO, « les Etats-membres s'engagent à la reconnaissance de la promotion et de la protection des droits de l'homme et des peuples, selon les termes de la Charte africaine des droits de l'homme et des peuples de $1981 »$.

Cependant, la Cour de Justice de la CEAE a un jugement très progressif des droits de l'homme à son crédit bien que sa juridiction explicite dans ce domaine reste à mettre en œuvre. Aussi a-t-elle été suffisamment courageuse pour garantir le respect des droits fonda-

96 C'est nous qui mettons entre guillemets.

97 Jean Joss MILINGO ÉLLONG, Art. cit., pp. 29-30.

98 En ce sens, voir Joseph ISSA-SAYEGH, Introduction au traité et aux actes uniformes de l'OHADA », Ohadata, D-02-17.9, cité par Jean Joss MILINGO ÉLLONG, Art. cit., p. 30. 
mentaux des individus au titre du traité ${ }^{99}$. C'est à ce propos que Jean note que l'« EAC s'est dotée d'une Cour de justice de l'Afrique de l'Est composée de six juges investis d'une juridiction pour entendre toute question provenant d'un différend entre les Etats membres ou pour prononcer des violations des droits de l'homme ${ }^{100}$ et qu'elle a été la troisième communauté économique régionale en Afrique à se doter d'une Cour expressément ou implicitement destinée à promouvoir et à protéger les droits de l'homme après la CEDEAO et la SADC.

\section{CONCLUSION}

Voilà donc présenté le système africain de protection des droits de l'homme, en dehors du mécanisme pris en charge par la Cour africaine des droits de l'homme et des peuples. Celui reste, il faut le reconnaitre encore lacunaire, mais il est en pleine mutation et consolidation notamment à travers le mécanisme juridictionnel qu'incarne la Cour africaine des droits de l'homme et des peuples (CADHP) en général et celui mis en progressivement en place par les organisations sous-régionales africaines. Ces dernières ont voulu ainsi rendre effectif et opérationnel le mécanisme déjà mis en place au niveau continental à travers notamment l'OUA d'abord et l'UA ensuite, la Charte africaine des droits de l'homme et des peuples ainsi que la Commission africaine des droits de l'homme et des peuples. Il est simplement à remarquer qu'en dépit de quelques faiblesses intrinsèquement liées à l'action desdits mécanismes s'agissant de la protection et de la promotion des droits de l'homme dans le cadre de leur champ respectif, ces mécanismes restent aussi révélateurs de ce que le droit international africain s'affirme et se consolide de plus en plus en tant que « ordre juridique spécifique » au regard de l'ordre juridique international général dont il constitue toutefois l'une de ses manifestations.

\section{BIBLIOGRAPHIE}

Abdoulaye SOMA, « Le jeu des protocoles dans le processus juridique de construction d'une Cour africaine de protection des droits de l'homme », in Revue CAMES/SJP, N002/2015, 2015, pp.1 - 18.

Auguste MAMPUYA KANUNK'A TSHIABO, Emergence des Etats nouveaux et droit international, Kinshasa 1984.

99 Ainsi le cas des affaires comme James KATABAZI and 21 others $v$ Secretary General of the East African Community and Another (East African Court of Justice at Arusha, reference 1 of 2007, 1 November 2007, in http :www.saflii.org/ea/cases/EACJ/2007/3html, (consulté le 24 novembre 2017) et Mary Ariviza and Okotch Mondoh Versus The Autorney General of the Republic of Kenya and the Secretary General of the East African Community (1 december 2010, Application $n^{0}$ 3 of 2010, in http:www.worldcourts.com.eacj/eng/decisions/2010.1201 Ariviza v Attorney General Kenya.html (consulté le 24 novembre 2017).

100 Joseph GAHAMA, La Communauté d'Afrique de l'Est : une jeune organisation régionale promise à un bel avenir?, sine loci, sine data. 
BALINGENE KAHOMBO, Présentation du Traité de droit international public du professeur Auguste Mampuya : Regards croisés sur le régionalisme africain, in Recht in Afrika - Law in Africa Droit en Afrique, 19 (2016), pp. 209-239.

BOUTROS BOUTROS-GHALI, L'Organisation de l'unité africaine, Paris 1969.

BULA BULA, S., "L'arrêt du 25 novembre 2005 de la Cour d'Appel de Dakar relatif à l'exception d'incompétences dans l'extradition de M. Hussein Habré » In Revue de Droit africain, n³6, octobre 2005, pp. 301-315

DIOP Abdou KHADRE, « La Cour africaine des droits de l'homme et des peuples ou le miroir stendhalien du système africain de protection des droits de l'homme », in Cahiers de Droit, Vol. 55, n², Juin 2014, 529-555.

DIOP MAMADOU, F., « Plaidoyer pour l'accès direct des individus à la Cour africaine des droits de l'homme et des peuples et à la future Cour africaine de justice, des droits de l'homme et des peuples », in Revue du Droit public, №2-2016, pp.664-687.

François BORELLA, Le régionalisme africain et l'Organisation de l'unité africaine, Annuaire français de droit international, IX (1963).

François BUGNION, Le comité international de la croix rouge et la protection des victimes de la guerre, $2^{\mathrm{ème}}$ éd., Genève, CICR, 2000.

G. Gurvitch, L'idée de droit social, Sirey,1932, (reéd. 1972).

Jacques CHEVALLIER, L'ordre juridique, sine loci, sine data.

James T. GATHII, Chapter 17: Africa, in: Bardo Fassbender et Anne Peters (eds.), The History of International Law, Oxford 2012, pp. 407-428.

Jean Joss MILINGO ÉLLONG, Les conflits de normes en matière de droits fondamentaux: le cas de l'OHADA et de l'Union africaine, in, Bulletin de droit économique, Université Laval, (2014) 2 B.D.E..

J-L. ATANGANA AMOUGOU, «Avancées et limites du système africain de protection des droits de l'homme : la naissance de la Cour africaine des droits de l'homme et des peuples » in Droits fondamentaux, $\mathrm{n}^{\circ} 3$, janvier-décembre 2003 [en ligne], décembre 2003, <www.droitsfondamentaux.or g> (page consultée le 30 juin 2010), pp. 135-152.

Jon Woronoff, Organizing African Unity, Metuchen 1970; Van Chien Nguyen, Les politiques d'unité africaine, thèse de 3e cycle, Université de Paris V, 1973

Joseph ISSA-SAYEGH, « Introduction au traité et aux actes uniformes de l'OHADA », Ohadata, D-0217.9.

Joseph-Marie BIPOUN-WOUM, Droit international africain: problèmes généraux, règlement des conflits, Paris, 1970.

KABORE, Antoine., "Les juridictions sénégalaises avaient-elles besoin de chambres extraordinaires pour poursuivre et juger Hussein Habré? ", in Revue belge de Droit international, Bruyant, Bruxelles, 2017, pp.90-111.

KAMGA Maurice., "Codification dans le domaine de la justice en Afrique ", in Journal of the African Union commission on international Law, 2ieme édition, Décembre 2015.pp. 324-398. 
KANDOLO ON'UFUKU wa KANDOLO, P.-F., Du système congolais de promotion et de protection des droits de l'homme. Contribution pour une mise en œuvre du mécanisme institutionnel spécialisé, Mémoire de D.E.A, Faculté de Droit, UNILU, 2011.

KATUALA KABA KASHALA (+), la preuve en Droit congolais (textes, jurisprudence et doctrine), Kinshasa, Batena Ntambwa,, 1998.

KAZADI MPIANA, J., « l'Union africaine face à la gestion des changements anticonstitutionnels de gouvernements, in Revue québécoise de droit international, Vol. 25.2., 2012, pp. 101-141.

KAZADI MPIANA, J., « La Protocole de Malabo face à la CPI : Concurrence ou complémentarité? Communication aux journées scientifiques de la Faculté de Droit de l'Université de Likasi, tenues de 15 mars 2017.pp. 1-5.

KAZADI MPIANA, J., « La saisine du juge africain des droits de l'homme par les individus et les ONG. Regards critiques sur les premiers arrêts et décisions de la Cour africaine des droits de l'homme et des peuples », In_Revue de droit international et de droit comparé, №2013, pp.315 - 352

KAZADI MPIANA, J., La position du Droit international dans l'ordre juridique congolais et l'application de ses normes, Paris, Publibook, 2013, pp..

KOAGNE ZOUAPET, A « L'Union Africaine à la recherche de son introuvable juridiction », sous la direction de MVELLE Guy et ZANG Laurent, l'Union Africaine quinze ans après, Tome 1, L'Harmattan, 2017, pp.279- 297.

Maia-OUMEIMA HAMROUNI, « La complémentarité régionale dans le cadre de la Cour africaine de justice, des droits de l'homme et des Peuples », in Revue du droit public, 2018, pp.1 - 14.

Maurice KAMTO., " La codification du droit international en Afrique : Méthode et défis », in Journal of the African Union Commission on international Law, 2ieme édition, Décembre 2015, 248- 268.

MAMPUYA KANUNK'a-TSHIABO, Traité de droit international public, MEDIASPAUL, KINSHASA, 2016.

MUBIALA MUTOY, "La Cour africaine des droits de l'homme et des peuples, dix ans ", in CongoAfrique, $\mathrm{N}^{\circ} 520$, Décembre 2017, pp.998 - 1001, pp.993- 1006.

MUBIALA MUTOY., "L'Afrique et la justice pénale internationale », In Revue internationale de droit pénal, Janvier, 2014, pp. 1-10.

MUSULWA SENGA, A., et MUYUMBA FUNDI., A., « Le désengagement des Etats africains de l'obligation de coopération avec la Cour Pénale Internationale : l'Union Africaine sur le banc des accusés », in Justitia, (Revue scientifique de la Faculté de Droit),Université de Lubumbashi, Lubumbashi, Mai,2018, pp. 225-245.

MUTOY MUBIALA, Le système régional africain de protection des droits de l'homme, coll. Organisation internationale et relations internationales, $\mathrm{n}^{0} 59$, Bruxelles, Bruylant, 2005.

NISRINE EBA NGUEMA, « La Commission africaine des droits de l'homme et des peuples et sa mission de protection des de l'homme " in la Revue des droits de l'homme, Revue du centre de recherche et d'études sur les droits fondamentaux, 11/2017, Varia, pp.1 - 16.

NTWARI Guy-F, « La Cour africaine des droits de l'homme et des peuples à la croisée des cheminsbilan des cinq premières années d'activités judiciaires (2009-2014), ATHENIS, In Revue trimestrielle, dr.h (102(2015),pp.368- 389. №520, Décembre 2017, pp.367- 391. 
Oswald NDESHYO, L'héritage de l'OUA à l'UA : la quête permanente de l'unité africaine, Kinshasa, 2010 Commission du droit international (CDI), 'Rapport de la Commission du droit international Cinquante-septième session (2 mai-3 juin and 11 juillet-5 août 2005)', Annuaire de la Commission $\mathrm{du}$ droit international II (2005), paras. 451-456.

Oswald NDESHYO (éd.), Manuel de droit communautaire africain. Tome I. Introduction générale : objet, sources, caractéristiques et domaines, Kinshasa 2011.

Pierre-François GONIDEC, Existe-t-il un droit international africain?, African Journal of International and Comparative Law, 5 (2) (1993), 243-259; Towards a 'treatise' of African international law', African Journal of International and Comparative Law, 9 (1997), 807-821

Pierre-François GONIDEC, Note sur le droit des conventions internationales en Afrique, Annuaire français de droit international, XI (1965), 879

Pierre-Marie DUPUY, P.-M. et KERBRAT, Y., Droit international public, Paris, 13ème éd. Dalloz, 2016.

SAIDOU NOUROU TALL, Droit du contentieux international africain, Dakar, L'Harmattan-Sénégal, 2018.

Stefaan SMIS et AMANI CIRIMWAMI, E, «Repenser la création fragmentée des juridictions hybrides en Afrique au profit de la Cour africaine de justice, des droits de l'homme et des peuples ", in Revue Belge de Droit international, Bruxelles, éd. Bruylant, 2017, pp.314 - 338.

TASLIM OLAWALE Elias, Africa and the Development of International Law, Leiden 1972 www.trial-ch.org/fr/ressources/commissions-verite/afrique/tchad.html, Consulté le 15 juillet 2018 à $14 \mathrm{~h} 33^{\prime}$. 